\title{
Identification of atherosclerosis-related prioritizing metabolites based on a multi-omics composite network
}

\author{
JUN-QIANG CAO ${ }^{1}$, CAI-XIA LI ${ }^{1}$, RU-YI WANG ${ }^{1}$, JIN-JIN CHEN ${ }^{2}$, \\ SHU-MEI MA ${ }^{1}$, WEN-YING WANG ${ }^{1}$ and LI-JUN MENG ${ }^{1}$
}

Departments of ${ }^{1}$ Cardiology and ${ }^{2}$ Anesthesiology, Binzhou City Center Hospital, Binzhou, Shandong 251700, P.R. China

Received May 28, 2018; Accepted January 31, 2019

DOI: $10.3892 / \mathrm{etm} .2019 .7351$

\begin{abstract}
Metabolites are the final products of cellular regulation processes, their level is the ultimate response of biological systems to environmental and genetic changes. Therefore, the identification of key metabolites is required for the diagnosis and therapy of diseases. In this study, atherosclerosis-related gene expression profile information was extracted from ArrayExpress database (GEOD-57691), and analyzed with limma package. Furthermore, we constructed an intricate multi-omics network involved in genes, phenotypes, metabolites and their associations. To identify the prioritization of atherosclerosis-related metabolites, the relation score of each metabolite in the composite network was computed with the random walk with restart (RWR) method. The top 50 metabolites and top 100 genes were chosen based on the score in the weighted composite network. Consequently, several key metabolites that were ranked in the top 5 of relation score or degree greater than 70 were confirmed. Particularly, metabolites Tretinoin and Estraderm not only have high relation scores, but also contain more degrees. Moreover, we obtained 24 co-expression genes that may be regarded as the targets of atherosclerosis therapy. Therefore, identification of metabolite prioritizations by the composite network integrated the information of genes, phenotypes and metabolites may be available to diagnose atherosclerosis, and can provide the potential therapeutic strategies for atherosclerosis.
\end{abstract}

\section{Introduction}

Atherosclerosis is the underlying cause of human death, which is involved in the onset of many diseases, including myocardial infarction, stroke, ischemic heart pain, and sudden cardiac death $(1,2)$. It has been widely reported that

Correspondence to: Dr Li-Jun Meng, Department of Cardiology, Binzhou City Center Hospital, 108 South Ring Road, Huimin, Binzhou, Shandong 251700, P.R. China

E-mail: menglijun112233@163.com

Key words: mRNA, atherosclerosis, metabolite, multi-omics composite network atherosclerosis mainly occurs in the intima of medium arteries at the blood flow disorganized regions and can be triggered by the interaction of endothelial dysfunction and subendothelial lipoprotein retention (3). A non-resolving inflammatory response is stimulated by the atherosclerotic triggering process and thus induces intimal destruction, end-organ ischemia and arterial thrombosis. Therefore, studies on the pathophysiology of atherosclerosis are urgently needed. Metabolomics has been reported to be used to identify the specific biomarkers and discover the possible metabolic pathways, which contributes to better exploration of the pathology of diseases.

Metabolites as the ultimate products of cellular regulatory process, are generally regarded as the final response of biological systems to genetic or environmental changes. It is well known that metabolite levels can be used to evaluate the current physiological state of human body. The identification of disease-associated metabolites has been demonstrated to be important for better understanding the pathophysiology of metabolites and enhancing the clinical diagnosis of diseases $(4,5)$. Metabolomics/metabonomics is a rapidly evolving field of biochemical research following genomics, transcriptomics and proteomics, which is used to quantitatively measure the metabolic response to pathophysiological stimuli. The study of metabolomics not only contributes to our understanding of the underlying molecular mechanisms of diseases, but also helps us for exploring the biomarkers of disease diagnosis $(6,7)$. Up to now, thousands of metabolites have been identified by metabolomics technologies, including gas chromatography-mass spectrometry (GC-MS), liquid chromatography-mass spectrometry (LC-MS), and nuclear magnetic resonance (NMR) (8). However, investigation of the identification and prioritization of high-risk metabolites associated with atherosclerosis are still a challenging task.

Generally, metabolites hardly function alone, and would be affected by genome and phenome when they are regarded as the link of genotypes and phenotypes. As known, one or two metabolites usually do not have a serious effect on the onset of a disease, but they diffuse among function-associated metabolites and genes organized into a complex network. Therefore, metabolites related to adjacent functions generally tend to be associated with phenotypically same or similar diseases (9). Furthermore, there are different functions for the metabolites in the network, and in the same functional module, some metabolites with strong correlation would 
play a special role in biological function. Currently, with the development of various 'omics' analysis, such as genomics, phenomics, and proteomics, it is beneficial to provide valuable information for prioritizing the high-risk metabolites associated with diseases. Therefore, a comprehensive and accurate information of disease-related metabolites can be provided by studying a multi-omics network involved in genes, metabolites and phenotypes.

In this study, an algorithm named MetPriCNet was used to predict the prioritization of atherosclerosis-related risk metabolites, which was performed by integrating multi-omics data. Yao et al have demonstrated that MetPriCNet not only has a high prediction validity in overall performance but also possesses an excellent identification ability of disease types (10). Consequently, a global metabolic network is involved in multi-omics network data, including phenotypic networks, genetic network, metabolic network and interaction network. Considering the global functional correlations of metabolites in this intricate network, the prioritization of key metabolites related to a certain phenotype can be confirmed. The freely accessible web of MetPriCNet is available at http:// www.bio-bigdata.net/MetPriCNet/.

\section{Materials and methods}

Obtaining multi-omics integrated information. A multi-omics composite network involved in genes, metabolites, phenotypes, gene-metabolite interactions, phenotype-gene interactions and phenotype-metabolite interactions was constructed. The data sources of relative information are described in the sections.

Gene network $\left(A_{G}\right)$. The human protein interacted network containing 1,048.576 interaction relationships were obtained from STRING (http://string-db.org/) database (11). We transformed the protein ID and gene name for removing the repetitive interaction relationships. Consequently, a PPI gene network with 16,785 nodes and 1,515.370 interaction relationships was obtained.

Metabolite network $\left(A_{M}\right)$. Firstly, 4,994 human metabolites were collected from the metabolite pathways of KEGG and HMDB databases, the human pathways of MSEA, SMPDB and Reactome databases (12-16). Subsequently, STITCH database was selected to collect the human metabolites and metabolite interaction relationships that were contained in the 4,994 human metabolites (17). Eventually, a metabolite network containing 3764 human metabolites and 74,667 metabolite interaction relationships was constructed.

Phenotype network $\left(A_{P}\right)$. We constructed the phenotype network containing 5,080 phenotypes that have similarity scores through applying the phenotype-phenotype similarity relationships from van Driel et al (18).

Gene-metabolite interaction networks $\left(A_{G M}\right)$. The chemicals, human genes and STITCH-associated information were extracted for obtaining the interaction between genes and metabolites. Based on the 4,994 human metabolites, we removed the genes that were not contained in the gene network and the metabolites that were not included in the metabolite network. Ultimately, a total of 192,763 gene-metabolite interactions involved in 12,342 genes and 3,278 metabolites were obtained.

Phenotype-gene interaction networks $\left(A_{G P}\right)$. The interactions between phenotypes and genes were collected from the Morbid Map file from OMIM. Similarly, the phenotypes that were not included in the phenotype network and the genes that were not involved in the gene network were removed. Consequently, we obtained 2,603 phenotype-gene interactions with 1,715 genes and 1,886 phenotypes. Notably, the weighted score of each phenotype-gene association was defined as 1 .

Phenotype-metabolite interaction network $\left(A_{P M}\right)$. The associations between phenotypes and metabolites can be extracted from HMDB database. Similarly, we removed the needless phenotypes and metabolites that were not found in the phenotype or metabolite network, respectively. Finally, 664 phenotype-metabolite associations containing 149 phenotypes and 388 metabolites were preserved.

Collecting disease-related gene expression data. Gene expression profiles associated with atherosclerosis were collected from ArrayExpress database (http://www.ebi. ac.uk/arrayexpress/) the access number is E-GEOD-57691. Then 10 samples called normal aortic tissue and 9 specimens named aortic atheroma tissue in expression sets were selected for further analysis. For improving the quality of the data, several standard pretreatments were performed, including probe correction, background correction, normalization and summarization of expressed value (19-21). As a result, an expression profile dataset containing 19,211 genes was obtained for subsequent analysis. The atherosclerosisassociated phenotype information with access number 143890 was obtained from Online Mendelian Inheritance in Man (OMIM; http://omim.org/) database. Integrating the gene expression profiles obtained from ArrayExpress and phenotype information containing related disease genes obtained from the Morbid Map file in OMIM database, the seed genes with differential expression were selected. To correctly screen the differentially expressed genes (DEGs), limma package was used to compute the differential expression (22). Moreover, t-test and F-test were conducted to analyze the gene expression matrix. Furthermore, $1 \mathrm{mFit}$ function was used to perform the empirical Bayes (eBayes) statistics and a false discovery rate (FDR) calibration of P-values for the data $(23,24)$. Eventually, we obtained the candidate genes with large difference for the establishment of the gene network.

Constructing an intricate multi-omics network. The six networks $\left(\mathrm{A}_{G}, \mathrm{~A}_{M}, \mathrm{~A}_{P}, \mathrm{~A}_{G M}, \mathrm{~A}_{G P}\right.$ and $\left.\mathrm{A}_{P M}\right)$ obtained above from multiple data sets were integrated into one weighted adjacency matrix A for constructing an intricate multi-omics network. Subsequently, based on the adjacency matrix, a column-normalized adjacency matrix $\mathrm{W}$ can be acquired, which is a transition matrix of the intricate network, $W_{i j}$ can be used to calculate the transition probability of each node from $\mathrm{i}$ to j. Ultimately, six networks were merged into a weighted composite network, and Yao et al have described the specific algorithm (10). 
Table I. The parameter of top 50 metabolites identified using the RWR method in the weighted composite network.

\begin{tabular}{|c|c|c|c|}
\hline Rank & Metabolite CID & Metabolite name & Score \\
\hline 1 & 444795 & Tretinoin $(\mathrm{TN})$ & 0.000677293 \\
\hline 2 & 5757 & Estraderm (TN) & 0.000622015 \\
\hline 3 & 5997 & Cholesterol (TN) & 0.000548486 \\
\hline 4 & 24139 & CHITIN & 0.000537016 \\
\hline 5 & 888 & Magnesium ion & 0.000473373 \\
\hline 6 & 6022 & Adenosine 5'-diphosphate & 0.000465706 \\
\hline 7 & 5280360 & Cervidil (TN) & 0.000462843 \\
\hline 8 & 5994 & Prometrium (TN) & 0.000450763 \\
\hline 9 & 5957 & Triphosadenine (DCF) & 0.000426371 \\
\hline 10 & 753 & Moctanin (TN) & 0.000415301 \\
\hline 11 & 945 & Nitric oxide & 0.000375328 \\
\hline 12 & 5280961 & Genistein & 0.00035226 \\
\hline 13 & 784 & Oxyfull (TN) & 0.000345901 \\
\hline 14 & 60823 & Lipitor (TN) & 0.00034207 \\
\hline 15 & 5280453 & Rocaltrol (TN) & 0.000339252 \\
\hline 16 & 923 & Sodium ion & 0.000331388 \\
\hline 17 & 172198 & Delivert (TN) & 0.000330823 \\
\hline 18 & 445154 & Resveratrol & 0.000317558 \\
\hline 19 & 77999 & Gaudil (TN) & 0.000314271 \\
\hline 20 & 444899 & Arachidonic acid & 0.000314223 \\
\hline 21 & 702 & Dehydrated ethanol & 0.000295643 \\
\hline 22 & 5280343 & Quercetin & 0.000294725 \\
\hline 23 & 3973 & 2-(4-Morpholinyl)-8-phenyl-4H-1-benzopyran-4-one & 0.000276657 \\
\hline 24 & 65064 & Epigallocatechin 3-gallate & 0.000273649 \\
\hline 25 & 312145 & Wortmannin & 0.000250849 \\
\hline 26 & 2336 & Benzo[a]pyrene & 0.000248994 \\
\hline 27 & 2244 & Acetylsalicylic acid & 0.000233603 \\
\hline 28 & 14985 & $\alpha$-Tocopherol & 0.000233033 \\
\hline 29 & 105024 & L-selenomethionine & 0.000229817 \\
\hline 30 & 445639 & Oleic acid (NF) & 0.00022218 \\
\hline 31 & 935 & Nickel & 0.000203525 \\
\hline 32 & 4829 & Pioglitazone (INN) & 0.000203337 \\
\hline 33 & 12035 & S-substitute & 0.000201323 \\
\hline 34 & 5816 & Adrenalin (TN) & 0.000197363 \\
\hline 35 & 24766 & Chondroitin 4-sulfate & 0.000196775 \\
\hline 36 & 439260 & Nor adrenalin (TN) & 0.000194196 \\
\hline 37 & 6076 & Cyclic AMP & 0.000189559 \\
\hline 38 & 5359597 & Superoxide anion & 0.000189271 \\
\hline 39 & 5280450 & Linoleic acid & 0.000188959 \\
\hline 40 & 774 & Histaminum (TN) & 0.000188733 \\
\hline 41 & 5460048 & (R)-triglyceride & 0.000187959 \\
\hline 42 & 158 & 14-hydroxy-E4-neuroprostane & 0.000184986 \\
\hline 43 & 778 & Homocysteine & 0.000183673 \\
\hline 44 & 977 & Oxygen (JP16/USP) & 0.000180497 \\
\hline 45 & 969516 & Curcumin & 0.000177388 \\
\hline 46 & 54687 & Pravator $(\mathrm{TN})$ & 0.000174654 \\
\hline 47 & 33032 & Gulutamine (USP) & 0.000173862 \\
\hline 48 & 23930 & Manganese & 0.000172384 \\
\hline 49 & 17756770 & Ceramide & 0.000172203 \\
\hline 50 & 985 & Palmitic acid (NF) & 0.000168691 \\
\hline
\end{tabular}


A

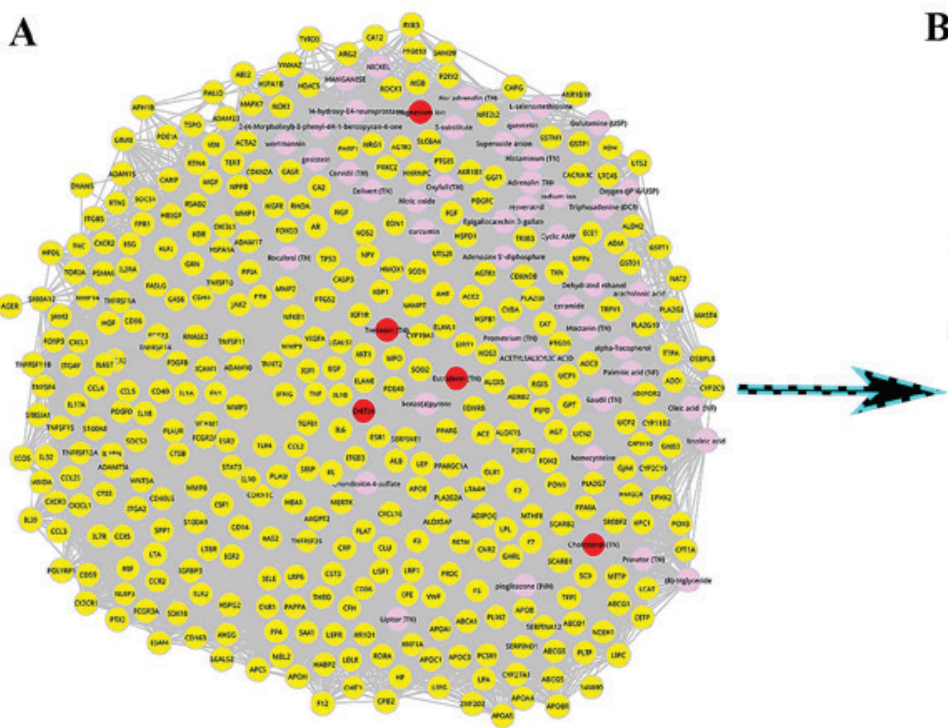

B

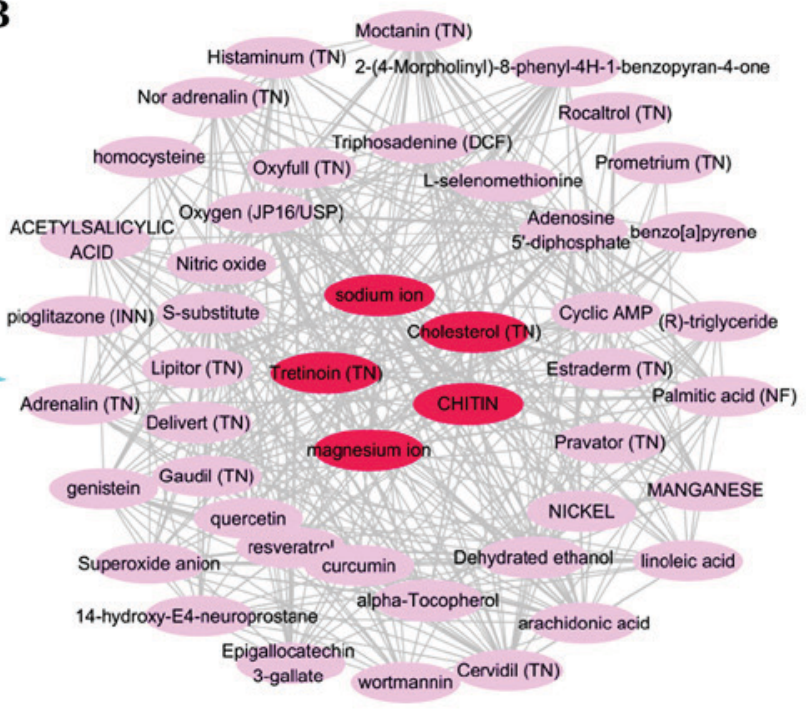

Figure 1. Prioritization of atherosclerosis-related metabolite network screened by multi-omics composite network. (A) Multi-omics composite network constructed by the atherosclerosis-related top 50 metabolites and seed genes. (B) Prioritizing metabolite network of atherosclerosis. Yellow, seed nodes, pink, the prioritizing metabolites, and red the top 5 metabolites.

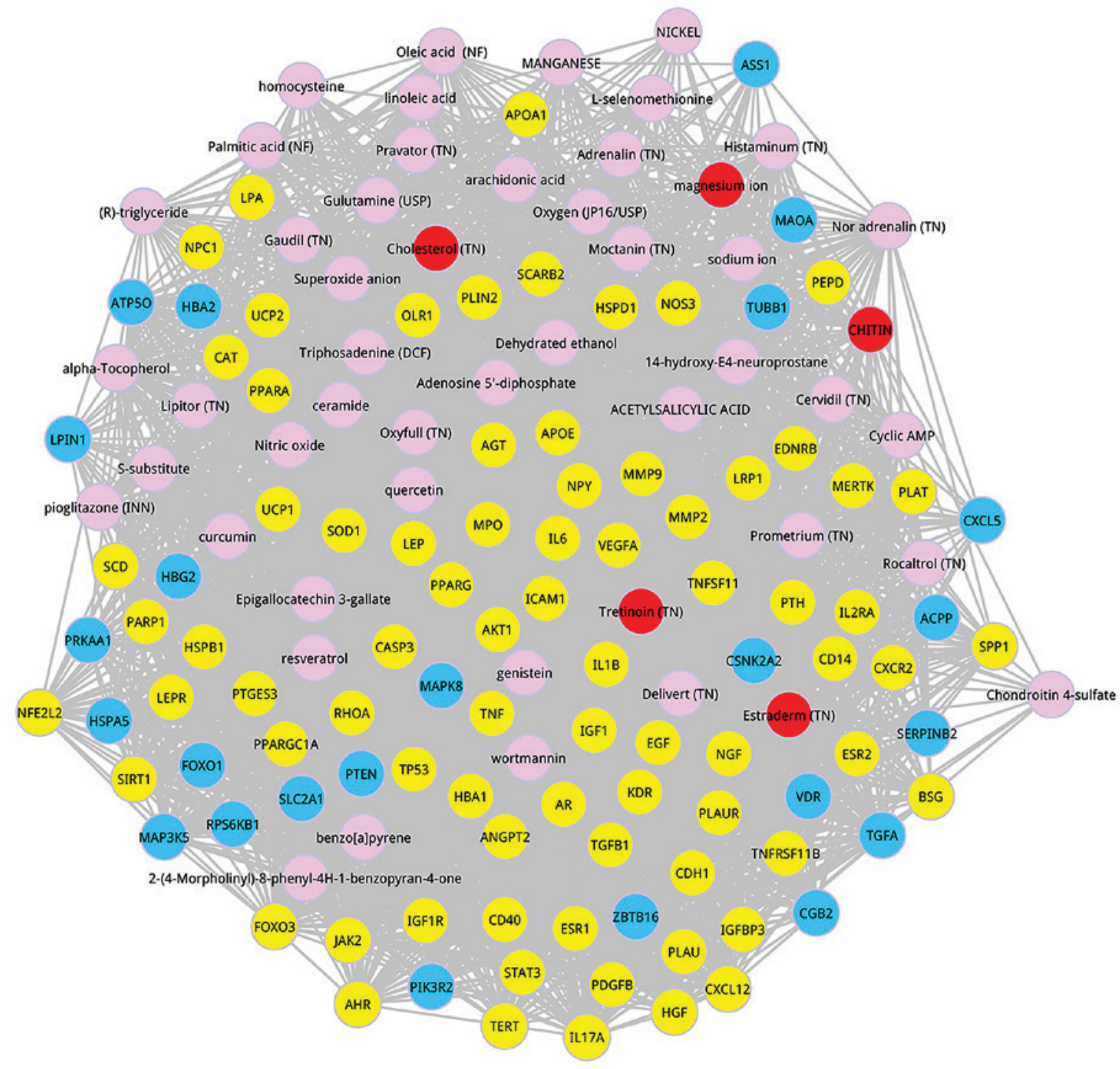

Figure 2. Co-expression network involved in top 50 metabolites, seed nodes and co-expression genes. Co-expression genes are marked in blue, metabolites are in pink, seed nodes are in yellow, and the top 5 metabolites are in red. 
Identification of candidate metabolite prioritization based on the weighted multi-omics composite network. To acquire the global omics information in the intricate network and to identify the prioritization of candidate metabolites, the random walk with restart (RWR) method was introduced into the intricate multi-omics network (25). On account of the proximity of each metabolite to the seed nodes, the prioritization of candidate metabolites was identified by the use of the RWR method, which was achieved through simulating the random walk processes of metabolites starting from a seed node. For each step, metabolite shifts from the current node(s) to its adjacent node(s) with probability $1-\alpha$ or goes back to the seed node(s) with probability $\alpha$. The probability is calculated as follows:

$$
P^{k+1}=(1-\alpha) W P^{k}+\alpha P^{0}
$$

Where $\mathrm{P}^{0}$ is the initial probability vector, $\mathrm{P}^{\mathrm{k}}$ is the probability vector that the $\mathrm{i}$-th element keeps in the node $\mathrm{i}$ at step $\mathrm{k}, \mathrm{W}$ is the transition matrix of the composite network. Notably, the obtained DEGs were used to provide the gene node in the intricate multi-omics network. It is worth mentioning that a stable probability calculated with the RWR method would be achieved by multiple iterations, and the probability reaches the steady state and terminates the iteration processes, as the change between $\mathrm{P}^{\mathrm{k}+1}$ and $\mathrm{P}^{\mathrm{k}}$ is lower than $10^{-10}$. Eventually, the relative probability of each metabolite in the composite network was obtained.

\section{Results}

The construction of an atherosclerosis-related composite multi-omics network. Atherosclerosis-related genes with larger differential expression were used to construct the gene network. In this study, we screened the atherosclerosis-related DEGs with limma package, and then applied t-test, F-test, eBayes statistics as well as FDR calibration. Ultimately, DEGs with the criteria of the adjusted $\mathrm{P}<0.05$ were selected for establishing the gene network. Furthermore, constructing an atherosclerosis-related composite multi-omics network containing the interactions of gene-gene, phenotype-phenotype, metabolite-metabolite, gene-phenotype, gene-metabolite and phenotype-metabolite by importing the gene network. Consequently, we obtained 9,993 nodes and 10,271.126 edges involved in atherosclerosis-related DEGs, phenotypes and metabolites.

Identifying the atherosclerosis-related metabolite prioritization. The prioritization of the atherosclerosis-related metabolites can be identified by evaluating the relation score of each metabolite in the composite network. Through combining the original weight score that was calculated with the RWR method in equation (1), the new relation score of each metabolite was computed, and further ranked the metabolite by the corresponding score. In the present study, we screened out 50 metabolites as the prioritizing candidate metabolites which ranked in the top 50 of relation score (Table I). Then, we searched for the prioritizing candidate metabolites from the multi-omics composite network, and thus extracting the atherosclerosis-related prioritizing metabolite composite
Table II. Co-expression genes associated with the top 50 metabolites of atherosclerosis in the multi-omics composite network.

\begin{tabular}{|c|c|c|c|}
\hline $\begin{array}{l}\text { Co-expression } \\
\text { gene }\end{array}$ & Degree & Closeness & adj.P.Val \\
\hline MAPK8 & 100 & 3.644899487 & 8.74E-05 \\
\hline PTEN & 81 & 3.526471283 & $2.45 \mathrm{E}-06$ \\
\hline FOXO1 & 59 & 2.841944445 & $2.20 \mathrm{E}-06$ \\
\hline SLC2A1 & 57 & 4.021206485 & $6.39 \mathrm{E}-05$ \\
\hline TGFA & 50 & 3.38473277 & $5.27 \mathrm{E}-05$ \\
\hline VDR & 47 & 3.99371055 & $3.87 \mathrm{E}-05$ \\
\hline HSPA5 & 46 & 2.673613247 & $8.11 \mathrm{E}-05$ \\
\hline CGB2 & 42 & 2.298998928 & $6.32 \mathrm{E}-05$ \\
\hline RPS6KB1 & 41 & 2.920705668 & $6.56 \mathrm{E}-05$ \\
\hline MAP3K5 & 41 & 3.568998598 & $1.67 \mathrm{E}-05$ \\
\hline HBG2 & 39 & 2.68166783 & 5.61E-05 \\
\hline ATP5O & 38 & 3.171190898 & $6.13 \mathrm{E}-06$ \\
\hline ACPP & 36 & 3.916740409 & $6.60 \mathrm{E}-05$ \\
\hline ZBTB16 & 35 & 2.764784562 & $1.44 \mathrm{E}-06$ \\
\hline PIK3R2 & 34 & 3.981092177 & $1.04 \mathrm{E}-04$ \\
\hline SERPINB2 & 33 & 3.450172722 & $7.11 \mathrm{E}-05$ \\
\hline PRKAA1 & 29 & 3.124904434 & $9.48 \mathrm{E}-05$ \\
\hline LPIN1 & 24 & 3.82077733 & $1.41 \mathrm{E}-06$ \\
\hline TUBB1 & 22 & 3.474172 & $9.47 \mathrm{E}-05$ \\
\hline CXCL5 & 21 & 3.173767298 & $1.91 \mathrm{E}-05$ \\
\hline HBA2 & 18 & 2.356349598 & 7.23E-05 \\
\hline ASS1 & 17 & 3.587780531 & 5.29E-06 \\
\hline MAOA & 15 & 3.373168305 & $3.44 \mathrm{E}-05$ \\
\hline CSNK2A2 & 13 & 3.148020812 & $3.90 \mathrm{E}-05$ \\
\hline
\end{tabular}

network. The associations of candidate metabolites are shown in Fig. 1, where the top 5 metabolites with higher score were marked in red, including Tretinoin $(\mathrm{TN})$ (score $=0.000677293)$, Estraderm (TN) (score $=0.000622015)$, Cholesterol $(\mathrm{TN})$ (score $=0.000548486$ ), CHITIN (score $=0.000537016$ ), magnesium ion $($ score $=0.000473373)$. Seed nodes were marked in yellow and other metabolites were in pink.

Identification of co-expression genes in the composite network. Based on the 50 prioritizing candidate metabolites, genes that were interacted with metabolites in the composite network were chosen. Furthermore, we analyzed the closeness between the known genes and candidate metabolites and screened out the co-expression genes, the closeness of which achieved a greater score than the average and ranked in top 100. Consequently, a co-expression network that was involved in the composite network was obtained (Fig. 2). In this network, yellow nodes represent the seed nodes, blue nodes represent the co-expression genes, pink nodes are the prioritizing candidate metabolites and red nodes are the top 5 metabolites. Ultimately, 24 co-expression genes marked in blue were identified, including 16 genes with degree greater than 30 and 8 genes with degree lower than 30 . The parameter information of the 24 co-expression genes is listed in Table II. Significantly, MAPK8 (also known as JNK1), that 
has the highest degree among those genes, was reported to be necessary for EC apoptosis and lipid deposition in the early atherogenesis (26). Furthermore, FOXO1 gene has been demonstrated to be expressed strongly in atherosclerotic plaques, and to participate in multiple atherogenic pathways of endothelial cells (27). By analyzing the degree of metabolites in the co-expression network, it can be found that 9 metabolites with degree more than 70 were confirmed, that is, Tretinoin (TN), Estraderm (TN), genistein, Oxyfull (TN), Dehydrated ethanol, quercetin, 2-(4-Morpholinyl)-8-phenyl-4H-1-benzopyran4-one, resveratrol and Nitric oxide. It is worth mentioning that Tretinoin (TN) was the metabolite that had the highest score, and Estraderm (TN) was the second metabolite followed by Tretinoin (TN).

\section{Discussion}

Atherosclerosis, a progressive disease, is the major cause of cardiovascular disease and stroke, and the leading cause of death worldwide. It generally occurs in the large arteries that accumulate a large number of lipids and fibrous elements (28). In the early lesions of atherosclerosis, numerous cholesterolengorged macrophages were accumulated in the subendothelial tissues, which is called 'foam cells'. The accumulation of lipids leads to more advanced lesions based on the foam cells. Plaques containing multiple compositions, such as calcification, fibrosis, cholesterol and hemorrhage, are formed, and thus lead to the acute occlusion of vessels. The pathogenesis of atherosclerosis is a complex and manifold process. The identification of disease-related metabolite prioritizations contributes to improving diagnosis of atherosclerosis-associated diseases, and helps exploring the metabolic pathological process.

Metabolites are the final products of cellular regulation processes, and can be used to assess the state of biological systems in genetic or environmental changes. As known, a metabolite is always affected by the combination of genes and phenotypes when it is considered as the link between genes and phenotypes. Furthermore, metabolites affected by one disease contain not only one or two, and some metabolites may play important biological functions in the development of a disease. Therefore, the identification of disease-related metabolite prioritization is necessary for improving the therapeutic effect of the disease. In this study, we integrated the multi-omics information that contains genes, metabolites, and phenotype related to pathogenesis, for predicting the atherosclerosisrelated metabolites. Moreover, the RWR method was used to compute the probability of each metabolite in the composite network for identifying the candidate metabolite prioritization. Consequently, several atherosclerosis-related key metabolites were confirmed, including the top 5 metabolites with high relation score [Tretinoin (TN), Estraderm (TN), Cholesterol (TN), Chitin and magnesium ion] and other 7 metabolites with high degree [Nitric oxide, genistein, Oxyfull (TN), resveratrol, Dehydrated ethanol, quercetin and 2-(4-Morpholinyl)-8phenyl-4H-1-benzopyran-4-one)].

Numerous studies indicate that antioxidants, such as vitamins $\mathrm{A}$ and E, can decrease the severity of atherosclerosis-related hypercholesterolemia and coronary heart disease (29,30). Significantly, Tretinoin (retinoic acid), a metabolite of vitamin A, plays a critical role in cell survival, differentiation and death, and has particular functions on neurogenesis and angiogenesis $(31,32)$. Tretinoin has been demonstrated to decrease the expression and activity of tissue factor in cultured human endothelial cells (33). Jiang et al have reported that Tretinoin can improve the atherogenic effects of $C$. pneumoniae by decreasing the damage of $C$. pneumoniae infection, and reduce the development of atherosclerotic lesions (34). Estraderm as a class of steroid hormone, is the most biologically active estrogen. Estrogen is reported to suppress the proliferation of vascular smooth muscle cells, the formation of atherosclerotic plaques, and oxidative stress. Investigation conducted by Li et al shows that low-concentration estrogen can function as an inhibitor for inhibiting the development of atherosclerosis through the mediation of cystathionine lyase-generated hydrogen sulfide (35). Furthermore, Dai et al demonstrated that estradiol and testosterone affect synergistically the early stage atherosclerosis, and estradiol/testosterone with an appropriate ratio can markedly inhibit the progression of atherosclerosis by decreasing foam cell formation, lipid lesions and endothelial damage (36). Additionally, the functional roles of other metabolites on the progression of atherosclerosis were also investigated, such as genistein suppress the atherosclerotic lesions by inhibiting the expression of matrix metalloproteinase-3 protein for protecting the vascular endothelial wall, Nitric oxide is regarded as a key modulator of vascular disease and the activation of endothelial nitric oxide synthase can improve atherosclerosis, and resveratrol functions as atheroprotective activity since the antioxidant, anti-inflammatory, antifibrotic, and cardioprotective properties (37-40).

In addition, we identified 24 co-expression genes that are closely related to the important atherosclerosis-associated metabolites. Moreover, some co-expression genes have been demonstrated to play a crucial role in the development of atherosclerosis, especially for genes with a high degree. For example, MAPK8 (degree: 100) as a member of the JNK family that play an important role in cardiovascular lesion and disease, has been reported to be required for apoptosis of endothelial cells and the deposition of lipids during early atherosclerosis $(26,41)$. Thus, inhibitors of MAPK8 may decrease atherosclerosis by reducing endothelial cell damage and preventing foam cell formation. PTEN gene (degree: 81) is reported to inhibit the expression of VCAM-1 molecule that is highly expressed in atherosclerotic lesions, suggesting that modulation of PTEN activity may contribute to reduce atherosclerosis (42). Furthermore, FOXO1 (degree: 59), a frequent transcription factor expressed frequently in mammalian cells, plays a crucial role in metabolic homeostasis regulation, cell differentiation, oxidative stress and autophagy $(43,44)$. Qiang et al studied the role of FOXO1 on atherosclerosis and endothelial cells, the results indicate that FOXO1 deacetylation can enhance the change of vascular endothelial cells, and thus contributing to the formation of atherosclerotic plaques (45). The functional effects of other co-expression genes on atherosclerosis are also reported, including HSPA5, CXCL5 and MAOA genes (46-48). Therefore, these results suggest that the metabolites and genes extracted by multi-omics composite network are important for the therapy of atherosclerosis.

In summary, atherosclerosis-related metabolites can be identified by the multi-omics composite network, and prioritization 
of metabolites can be confirmed by the use of the RWR method. Several key metabolites were found, including Tretinoin (TN), Estraderm (TN), Cholesterol (TN), Chitin, magnesium ion, Nitric oxide, genistein, Oxyfull (TN), resveratrol, dehydrated ethanol, quercetin and 2-(4-Morpholinyl)-8-phenyl-4H-1benzopyran-4-one, which could be considered as biomarkers of atherosclerosis diagnosis. Furthermore, 24 co-expression genes, identified by analyzing the metabolites in composite network, may be available to be targets of atherosclerosis therapy. However, for the analysis of metabolites and genes, some limitations are still present. Firstly, there are many diseases associated with atherosclerosis, whereas we only selected one dataset (GEOD-57691). Additionally, the candidate metabolites extracted by the multi-omics composite network have not been verified by clinical experiments. Nonetheless, the results obtained from this study are still able to provide some preliminary evidence to explore the potential therapeutic strategies for atherosclerosis.

\section{Acknowledgements}

Not applicable.

\section{Funding}

No funding was received.

\section{Availability of data and materials}

The datasets used and/or analyzed during the current study are available from the corresponding author on reasonable request.

\section{Authors' contributions}

JQC, CXL and SMM performed the experiments and analyzed the data. JQC was also a major contributor in writing the manuscript. RYW and JJC made a substantial contribution to the study concept and design. JQC, CXL and WYW performed the statistical analysis. LJM was involved in the conception and design of the study as well as the drafting of the manuscript. All authors read and approved the manuscript.

\section{Ethics approval and consent to participate}

Not applicable.

\section{Patient consent for publication}

Not applicable.

\section{Competing interests}

The authors declare that they have no competing interests.

\section{References}

1. Lusis AJ: Atherosclerosis. Nature 407: 233-241, 2000.

2. Akerele OA and Cheema SK: Fatty acyl composition of lysophosphatidylcholine is important in atherosclerosis. Med Hypotheses 85: 754-760, 2015.
3. Tabas I, García-Cardeña G and Owens GK: Recent insights into the cellular biology of atherosclerosis. J Cell Biol 209: 13-22, 2015.

4. Holmes E, Wilson ID and Nicholson JK: Metabolic phenotyping in health and disease. Cell 134: 714-717, 2008.

5. Iqbal O, Ottman A, Gaynes J, De Alba F, Gaynes BI, Fareed J and Bouchard CS: The effects of aspirin and its metabolites on peripheral blood mononuclear cells and human retinal pigment epithelial cells - implications in the pathophysiology of agerelated macular degeneration. IOVS 55: 634, 2014.

6. Kell DB: Metabolomics and systems biology: Making sense of the soup. Curr Opin Microbiol 7: 296-307, 2004.

7. Nicholson JK and Lindon JC: Systems biology: Metabonomics. Nature 455: 1054-1056, 2008

8. Moco S, Bino RJ, De Vos RCH and Vervoort J: Metabolomics technologies and metabolite identification. Trends Analyt Chem 26: 855-866, 2007.

9. Lee DS, Park J, Kay KA, Christakis NA, Oltvai ZN and Barabási AL: The implications of human metabolic network topology for disease comorbidity. Proc Natl Acad Sci USA 105: 9880-9885, 2008.

10. Yao Q, Xu Y, Yang H, Shang D, Zhang C, Zhang Y, Sun Z, Shi X, Feng L, Han J, et al: Global prioritization of disease candidate metabolites based on a multi-omics composite network. Sci Rep 5: 17201, 2015.

11. von Mering C, Huynen M, Jaeggi D, Schmidt S, Bork P and Snel B: STRING: A database of predicted functional associations between proteins. Nucleic Acids Res 31: 258-261, 2003.

12. Wishart DS, Knox C, Guo AC, Eisner R, Young N, Gautam B, Hau DD, Psychogios N, Dong E, Bouatra S, et al: HMDB: A knowledgebase for the human metabolome. Nucleic Acids Res 37: D603-D610, 2009.

13. Kanehisa M, Goto S, Sato Y, Furumichi M and Tanabe $M$ KEGG for integration and interpretation of large-scale molecular data sets. Nucleic Acids Res 40: D109-D114, 2012.

14. D'Eustachio P: Reactome knowledgebase of human biological pathways and processes. Methods Mol Biol 694: 49-61, 2011

15. Xia J and Wishart DS: MSEA: a web-based tool to identify biologically meaningful patterns in quantitative metabolomic data. Nucleic Acids Res 38 (Suppl 2): W71-W77, 2010.

16. Jewison T, Su Y, Disfany FM, Liang Y, Knox C, Maciejewski A, Poelzer J, Huynh J, Zhou Y, Arndt D, et al: SMPDB 2.0: Big improvements to the Small Molecule Pathway Database. Nucleic Acids Res 42: D478-D484, 2014

17. Kuhn M, Szklarczyk D, Franceschini A, Campillos M, von Mering C, Jensen LJ, Beyer A and Bork P: STITCH 2: An interaction network database for small molecules and proteins. Nucleic Acids Res 38 (Suppl 1): D552-D556, 2010.

18. van Driel MA, Bruggeman J, Vriend G, Brunner HG and Leunissen JA: A text-mining analysis of the human phenome. Eur J Hum Genet 14: 535-542, 2006.

19. Bolstad BM, Irizarry RA, Åstrand M and Speed TP: A comparison of normalization methods for high density oligonucleotide array data based on variance and bias. Bioinformatics 19: 185-193, 2003.

20. Irizarry RA, Bolstad BM, Collin F, Cope LM, Hobbs B and Speed TP: Summaries of Affymetrix GeneChip probe level data. Nucleic Acids Res 31: e15, 2003.

21. Belitskaya-Lévy I, Zeleniuch-Jacquotte A, Russo J, Russo IH, Bordás P, Ahman J, Afanasyeva Y, Johansson R, Lenner P, Li X, et al: Characterization of a genomic signature of pregnancy identified in the breast. Cancer Prev Res (Phila) 4: 1457-1464, 2011.

22. Ritchie ME, Phipson B, Wu D, Hu Y, Law CW, Shi W and Smyth GK: limma powers differential expression analyses for RNA-sequencing and microarray studies. Nucleic Acids Res 43: e47, 2015.

23. Reiner A, Yekutieli D and Benjamini Y: Identifying differentially expressed genes using false discovery rate controlling procedures. Bioinformatics 19: 368-375, 2003.

24. Datta S, Satten GA, Benos DJ, Xia J, Heslin MJ and Datta S: An empirical bayes adjustment to increase the sensitivity of detecting differentially expressed genes in microarray experiments. Bioinformatics 20: 235-242, 2004.

25. Wu X, Jiang R, Zhang MQ and Li S: Network-based global inference of human disease genes. Mol Syst Biol 4: 189, 2008.

26. Amini N, Boyle JJ, Moers B, Warboys CM, Malik TH, Zakkar M, Francis SE, Mason JC, Haskard DO and Evans PC: Requirement of JNK1 for endothelial cell injury in atherogenesis. Atherosclerosis 235: 613-618, 2014. 
27. Kedenko L, Lamina C, Kedenko I, Kollerits B, Kiesslich T, Iglseder B, Kronenberg F and Paulweber B: Genetic polymorphisms at SIRT1 and FOXO1 are associated with carotid atherosclerosis in the SAPHIR cohort. BMC Med Genet 15: 112, 2014.

28. Hadi NR: Effect of vildagliptin on atherosclerosis progression in high cholesterol - fed male rabbits. J Clin Exp Cardiolog 04: 15025,2016

29. Gey KF and Puska P: Plasma vitamins E and A inversely correlated to mortality from ischemic heart disease in crosscultural epidemiology. Ann NY Acad Sci 570: 268-282, 1989.

30. Hornig B: Vitamins, antioxidants and endothelial function in coronary artery disease. Cardiovasc Drugs Ther 16: 401-409, 2002.

31. Környei Z, Gócza E, Rühl R, Orsolits B, Vörös E, Szabó B, Vágovits B and Madarász E: Astroglia-derived retinoic acid is a key factor in glia-induced neurogenesis. FASEB J 21: 2496-2509, 2007.

32. Bowles J, Secker G, Nguyen C, Kazenwadel J, Truong V, Frampton E, Curtis C, Skoczylas R, Davidson TL, Miura N, et al: Control of retinoid levels by CYP26B1 is important for lymphatic vascular development in the mouse embryo. Dev Biol 386: 25-33, 2014.

33. Kim YM, Kim JH, Park SW, Kim HJ and Chang KC: Retinoic acid inhibits tissue factor and HMGB1 via modulation of AMPK activity in TNF- $\alpha$ activated endothelial cells and LPS-injected mice. Atherosclerosis 241: 615-623, 2015.

34. Jiang SJ, Campbell LA, Berry MW, Rosenfeld ME and Kuo CC: Retinoic acid prevents Chlamydia pneumoniae-induced foam cell development in a mouse model of atherosclerosis. Microbes Infect 10: 1393-1397, 2008.

35. Li H, Mani S, Wu L, Fu M, Shuang T, Xu C and Wang R: The interaction of estrogen and $\mathrm{CSE} / \mathrm{H} 2 \mathrm{~S}$ pathway in the development of atherosclerosis. Am J Physiol Heart Circ Physiol 312: H406-H414, 2017.

36. Dai W, Ming W, Li Y, Zheng HY, Wei CD, Rui Z and Yan C: Synergistic effect of a physiological ratio of estradiol and testosterone in the treatment of early-stage atherosclerosis. Arch Med Res 46: 619-629, 2015.

37. Lee CS, Kwon SJ, Na SY, Lim SP and Lee JH: Genistein supplementation inhibits atherosclerosis with stabilization of the lesions in hypercholesterolemic rabbits. J Korean Med Sci 19: 656-661, 2004

38. Voloshyna I, Hussaini SM and Reiss AB: Resveratrol in cholesterol metabolism and atherosclerosis. J Med Food 15: 763-773, 2012.
39. Tin A, Grams ME, Maruthur NM, Astor BC, Couper D, Mosley TH, Selvin E, Coresh J and Kao WH: Results from the Atherosclerosis Risk in Communities study suggest that low serum magnesium is associated with incident kidney disease. Kidney Int 87: 820-827, 2015.

40. Förstermann U, Xia N and Li H: Roles of vascular oxidative stress and nitric oxide in the pathogenesis of atherosclerosis. Circ Res 120: 713-735, 2017.

41. Babaev VR, Yeung M, Erbay E, Ding L, Zhang Y, May JM, Fazio S, Hotamisligil GS and Linton MF: Jnk1 deficiency in hematopoietic cells suppresses macrophage apoptosis and increases atherosclerosis in low-density lipoprotein receptor null mice. Arterioscler Thromb Vasc Biol 36: 1122-1131, 2016.

42. Yuan M, Wang X, Zhan Q, Duan X, Yang Q and Xia J: Association of PTEN genetic polymorphisms with atherosclerotic cerebral infarction in the Han Chinese population. J Clin Neurosci 19: 1641-1645, 2012.

43. Accili D and Arden KC: FoxOs at the crossroads of cellular metabolism, differentiation, and transformation. Cell 117: 421-426, 2004

44. Sengupta A, Molkentin JD and Yutzey KE: FoxO transcription factors promote autophagy in cardiomyocytes. J Biol Chem 284: 28319-28331, 2009.

45. Qiang L, Tsuchiya K, Kim-Muller JY, Lin HV, Welch C and Accili D: Increased atherosclerosis and endothelial dysfunction in mice bearing constitutively deacetylated alleles of Foxol gene. J Biol Chem 287: 13944-13951, 2012.

46. Zhao J, Forsberg CW, Goldberg J, Smith NL and Vaccarino V: MAOA promoter methylation and susceptibility to carotid atherosclerosis: Role of familial factors in a monozygotic twin sample. BMC Med Genet 13: 100, 2012.

47. Rousselle A, Qadri F, Leukel L, Yilmaz R, Fontaine JF, Sihn G, Bader M, Ahluwalia A and Duchene J: CXCL5 limits macrophage foam cell formation in atherosclerosis. J Clin Invest 123: 1343-1347, 2013.

48. Orešič M, Clish CB, Davidov EJ, VerheijE, Vogels J, Havekes LM, Neumann E, Adourian A, Naylor S, van der Greef J and Plasterer T: Phenotype characterisation using integrated gene transcript, protein and metabolite profiling. Applied Bioinformatics 3: 205-217, 2004.

This work is licensed under a Creative Commons Attribution-NonCommercial-NoDerivatives 4.0 International (CC BY-NC-ND 4.0) License. 